\title{
Décrochage Et Vécu Scolaires Des Élèves Autochtones De La Circonscription Scolaire De Zanaga, République Du Congo
}

\author{
${\text { Yvette BakinguBakibangou }{ }^{1 *} \text {, Samuel Mawete }}^{2}$ \\ ${ }^{I}$ Docteur en Sciences de l'Education, Enseignante chercheure à l'Institut Supérieur d'Education Physique et \\ Sportive (Université Marien Ngouabi) \\ ${ }^{2}$ Professeur en Sciences de l'Education Ecole Normale Supérieur (Université Marien Ngouabi)
}

*Corresponding Author: Yvette BakinguBakibangou, Docteur en Sciences de l'Education, Enseignante chercheure à l'Institut Supérieur d'Education Phvsique et Sportive (Université Marien Ngouabi)

\begin{abstract}
This paper purports to investigate the phenomenon of early school dropout among aboriginal children and ask for their opinions concerning their school experience, with a view to suggesting avenues of intervention favorable to their retention in school. This studyhas been conducted through quantitative and qualitative approaches. As a result, data have been collected from school re-entry reports of nine primary schools in Zanaga and from interviews with eleven aboriginal dropout students. The results obtained showthat dropout rates remain high, despite the retention policies in place. The school life is estimated at two years. Also, the schooling of these students has been marked by rejection, discrimination and maladjustment to educational programs. In order to ensure the educational sustainability of these students, a synergy of actions is to be recommended, in particular promoting policies of living together between different human groups, integrating elements of indigenous culture into educational programs and preparing for their integration in formal schools, as experiencedby 'ORA schools' initiated in Sangha and Likouala Departments.
\end{abstract}

Keywords: Aboriginal, dropping out, staying in school, early learning cycle, primary school

\section{INTRODUCTION}

Depuis l'adoption de la déclaration universelle des droits de l'homme en 1948, l'éducation est reconnue comme un droit pour tous. Dans le même sens, la convention des Nations Unies de 1989, relative aux droits de l'enfant a renforcé et élargi davantage le concept de droit à l'éducation, en instituant particulièrement l'obligation de prendre en compte dans les politiques éducatives : les principes de la non-discrimination, l'intérêt supérieur de l'enfant, le droit à la vie et à la survie, le développement de l'enfant dans toute la mesure du possible et le droit de l'enfant à exprimer librement ses opinions. (ONU, 1989).

Grâce à ces instruments juridiques sus mentionnés, au lendemain de la conférence de l'éducation de Dakar tenue en 2000, de nombreux pays ont défini et mis en œuvre des politiques en faveur de la promotion de l'éducation pour tous. En conséquence, des progrès significatifs ont été enregistrés concernant la scolarisation. Toutefois, beaucoup reste à faire, surtout dans les pays africains où les discriminations d'ordre, sociétal et scolaire persistent encore.

Le système éducatif congolais n'est pas épargné de cette réalité. En effet, selon le rapport de l'Institut National de la Statistique de 2014, le taux net de scolarisation a atteint $80 \%$. Cet indicateur de performance quoique significatif, est malheureusement encore loin d'être satisfaisant, puisque la visibilité des enfants autochtones sur la scène éducative est encore très faible. A propos, KamaNiamayoua (2008), faisant l'état des lieux sur la scolarisation des enfants autochtones, dans sa communication à la $48^{\text {ème }}$ session de la conférence internationale de l'éducation, a rapporté que 12 857 enfants étaient scolarisés, soit un pourcentage d'environ 6\%. En plus elle a relevé des écarts considérables entre les effectifs des élèves inscrits au Cours Préparatoire première année (CP1) et ceux du Cours Moyen deuxième année (CM2). Dans le département de la Lékoumou par exempledeux cent soixante-seize(276) élèves étaient inscrits au CP1 et un (01) seulement au CM2. C'est donc à la lumière de ces indicateurs de performances que notre réflexion prend toute sa signification. 
Il est vrai que la littérature portant sur les obstacles à la scolarisation des enfants autochtones est abondante, mais la question du décrochage et du vécu scolaire de ces enfants est peu explorée dans le paysage éducatif congolais. C'est dans ce contexte que s'est construit notre thème de recherche intitulé: Décrochage et vécu scolaires des élèves autochtones de la circonscription scolaire de Zanaga, en République du Congo.

Nous sommes certains que ce travail de recherche ne sera pas sans intérêt. Nous présumons que les résultats qui en découleront, permettront, au plan scientifique, de vérifier l'hypothèse selon laquelle la configuration de l'école aurait des effets sur le décrochage scolaire. Aussi, ils contribueront à faire avancer les connaissances concernant les facteurs susceptibles de favoriser l'adaptation des élèves à risque de décrochage scolaire, issus des populations socioéconomiques défavorisées.

Au plan social, la pertinence de cette étude est à situer à deux niveaux. D'une part, elle permettra d'identifier les facteurs scolaires à l'origine du décrochage chez l'enfant autochtone et de définir les stratégies efficientes de maintien à l'école. D'autre part, la diffusion des résultats issus de ce travail de recherche permettra de sensibiliser les enseignants, quant au rôle qu'ils peuvent jouer dans le soutien à la persévérance scolaire de cette catégorie d'élèves.

Il est de fait qu'en République du Congo l'éducation a toujours été considérée comme une priorité des priorités. Depuis l'an 2000 et conformément aux exigences de la conférence de l'éducation de Dakar (EPT), elle a souscrit à l'engagement de faire en sorte que tous les enfants où qu'ils trouvent sur le du territoire national, sans discrimination, soient scolarisés et qu'ils terminent au moins le cycle primaire, conformément aux objectifs de l'Education Pour Tous (EPT).

Afin de réaliser cette ambitieuse vision, elle a pris certaines mesures, parmi lesquelles la promulgation en 2007 de la loi portant la gratuité des frais de scolarisation pour tous les élèves qui fréquentent les écoles primaires publiques. Au cours de la même année, pour booster la scolarité et maintenir les enfants à l'école, les pouvoirs publics avec l'appui des institutions internationales: le PAM, l'UNICEF et les Organisations Non Gouvernementale (ONG) ont institué les cantines scolaires dans les zones les plus défavorisées. Les élèves autochtones, partageant le même espace d'apprentissage scolaire avec les élèves Bantous de facto bénéficient de ces politiques incitatives.

Seulement, s'il y a une réalité qu'on ne peut contester, c'est bien la constatation incessante du décrochage scolaire chez les élèves autochtones. Cette réalité a suscité un certain nombre de questions que nous nous proposons de répondre dans ce travail de recherche.

- Quelle est la situation actuelle du décrochage scolaire des élèves autochtones dans les écoles de la circonscription scolaire de Zanaga?

- Si nous admettons avec Janosz et al., (op.cit.) que l'expérience scolaire négative est la variable la plus prédictive du décrochage scolaire, quelles ont été les faits saillants dans l'expérience scolaire des décrocheurs autochtones?

Conséquemment à ce questionnement, les hypothèses qui sous-tendent ce travail de recherche sont définies comme suit:

- Le décrochage scolaire des élèves autochtones dans les écoles de la circonscription scolaire de Zanaga serait toujours préoccupant;

- L'expérience scolaire des décrocheurs autochtones serait empreinte de relations négatives et d'inadaptation au programme d'enseignement/apprentissage.

Ce travail de recherche vise les objectifs suivants:

$\checkmark$ faire un état des lieux du décrochage scolaire des enfants autochtones dans les écolesde la circonscription scolaire de Zanaga ;

$\checkmark$ requérir les opinions des décrocheurs autochtones concernant leur vécue scolaire.

Notre travail de recherche est structuré en quatre parties hormis l'introduction et la conclusion. Il s'agit, respectivement du cadre théorique, la méthodologie, les résultats obtenus et la discussion.

\section{CONSIDÉRATIONS THÉORIQUES DE L'ÉTUDE}

Notre analyse se construit autour de deux concepts clés: élèves autochtones et décrochage scolaire. Afin de rendre nos propos compréhensibles, un éclairage sémantique de ces concepts est indispensable. 
Décrochage Et Vécu Scolaires Des Élèves Autochtones De La Circonscription Scolaire De Zanaga, République Du Congo

Le terme autochtone vient du grec autokhthôn, à savoir « Autos » qui signifie «soi-même » et de « Khthôn » qui renvoie à la «terre ». Partant de cette acception étymologique, le terme autochtone est utilisé pour désigner la personne qui est issue du sol même où il habite, en opposition à celle qui vient d'ailleurs (l'immigré).

Dans la littérature, plusieurs termes sont utilisés pour désigner les populations autochtones. Ces désignations varient en fonction des pays. En Chine, la « minorité ethnique » correspond à ce que l'on considère ailleurs comme un peuple autochtone. En Inde, on parle de «tribu répertoriée » ou Adivasi (les premiers habitants). Au nord de la Scandinavie et de certaines régions de la Russie, on trouve les Samis. En Afrique, les populations autochtones sont désignées à partir de leurs modes de vie et de subsistance socioéconomique. On parle par exemple de pastoralisme, de chasseurs-cueilleurs, de pêcheurs, d'agriculteurs. Enfin, en République du Congo, cette communauté minoritaire est parfois désignée par le vocable 'pygmée'.

En ce qui concerne la situation démographique des peuples autochtones, il est important de rappeler qu'ilssont répartis dans plus de 90 pays dans le monde. Selon les estimations du rapport des Nations Unies (2014), ces peuples représentaient environ 370 millions de personnes dans le monde, soit 5\% de la population mondiale. En République du Congo, la population autochtone est estimée à 84783 , soit $3 \%$ de la population nationale. Elle est principalement concentrée dans trois départements, notamment, la Likouala, la Lékoumou et la Sangha, où elle représente près de $76 \%$ de leur effectif total. (Kitsimbou, 2020).

Par ailleurs, malgré la reconnaissance de leurs droits, tant au niveau national qu'international (ONU, 1959; UNESCO, 1994; Loi n5-2011), de manière générale, les peuples autochtones, où qu'ils vivent, sont encore confrontés à d'énormes difficultés et figurent systématiquement dans les catégories sociales des plus pauvres et des plus marginalisés. En effet, ils rencontrent les difficultés dans les domaines tels que la culture, l'éducation, la santé, les droits de l'homme, dans le développement économique et social.

Dans le domaine éducatif, l'égalité d'accès est restée un slogan dans bien des systèmes éducatifs. En République du Congo, les enfants autochtones sont peu visibles sur la scène éducative, alors qu'ils font pourtant partie pleinement de la population scolaire congolaise. Aussi, de nombreux travaux de recherche (Presseau et al.,2006 ; Mafoukila, 2006 et Itoua-Yoyo 2008) portant sur les parcours scolaires des élèves autochtones, ont montré que ceux- sont atypiques, c'est-à-dire, marqués par des échecs, des ruptures, un rythme de fréquentation scolaire saisonnier...Aussi,Kono et al., (2013, p.5) ont déclaré qu': " on trouve peu de jeunes peuples autochtones ayant obtenu des diplômes de l'enseignement primaire et secondaire ».

Les résultats de ces études révèlent que ces élèves autochtones quittent l'école avant d'avoir terminé un cycle scolaire amorcé. En d'autrestermes, ils décrochent.

Pour traduire le refus ou l'abandon scolaire par les élèves, on emploie plusieurs termes, par exemple, Glasman (2011) parle de "démobilisation scolaire", et de "décrochage scolaire". Ballion, (2000) utilise plutôt le terme "désengagement scolaire". Pour des raisons de préférence linguistique, dans ce travail de recherche nous privilégions le terme de" décrochage scolaire". Selon Glasman (ibid) le décrochage scolaire est le nouveau nom de l'échec scolaire. Blaya, et al. (2003) ont choisi le terme décrochage pour désigner le processus de désadhésion d'un élève ausystème ou un accrochage manqué qui conduira à plus ou moins long terme à une désaffection de l'école. Dans la même optique, Favresse et al., (2004) le considèrent comme étant la conséquence de la détérioration du lien entre le jeune, l'école et la société.

De nombreuses études (Blaya, 2010a; Blaya, 2010b; Douat, 2011; Fortin et al., 2004) ont montré que les causes du décrochage sont multifactorielles, notamment le climat scolaire, les comportements agressifs, la dépression, le déficit d'attention, le climat familial...

Si tous ces facteurs ont leur importance, néanmoins, il n'en demeure pas moins que les problèmes rencontrés par les élèves à l'école constituent l'un des facteursles plus déterminants. A propos, Janosz et al., (2000) pensent que l'expérience scolaire négative est la variable la plus prédictive du décrochage scolaire. Dans le même sens, Lee et al., (2003) laissent entendre que l'environnement scolaire global semble jouer un rôle dans le risque de l'abandon, spécifiquement lorsque l'école n'offre pas aux élèves un cadre clair, structuré et cohérent, ou lorsque le contexte scolaire est dégradé. 
Décrochage Et Vécu Scolaires Des Élèves Autochtones De La Circonscription Scolaire De Zanaga, République Du Congo

De ce qui précède, notre analyse du décrochage se focalise sur l'environnement scolaire, plus précisément sur les relations interpersonnelles, les attitudes...

Par ailleurs, l'ancrage théorique sur lequel s'appuie notre étude est la théorie des systèmes de Bronfenbrenner (1993). Cette théorie repose sur le postulat selon lequel: l'environnement, au sens large, influence le développement de l'enfant. Aussi, de la qualité de gestion de ces interactions dépendra la qualité du développement de l'enfant. Dans le cas d'espèce, nous voulons analyser le décrochage scolaire des enfants autochtone en rapport avec leur expérience scolaire.

\section{MÉTHOdologie}

Pour atteindre les objectifs définis, nous avons combiné deux approches: l'une quantitative et l'autre qualitative. L'approche quantitative a consisté en une analyse descriptive du phénomène à partir des effectifs des élèves autochtones inscrits dans les écoles primaires de la circonscription scolaire de Zanaga. L'approche qualitative, quant à elle, a consisté à analyser les opinions des décrocheurs autochtones, concernant leur vécu scolaire, en vue de déceler les obstacles à la scolarisation chez cette catégorie d'élèves.

En ce qui concerne les instruments mobilisés pour la collecte des données, en tenant compte de la nature de l'étude, nous avons privilégié la recherche documentaire et l'entrevue. La recherche documentaire a consisté à consulter les rapports de rentrée et de relever les effectifs des élèves autochtones inscrits dans ces écoles. Il sied de préciser que nous avons considéré les données disponibles, de trois années scolaires, notamment de 2017 à 2020. Pour la recherche qualitative un guide d'entretien a servi de support aux entrevues. Celui-ci a été constitué de deux thèmes suivants : considération de l'école et les rapports à l'école.

L'étude s'est réalisée dans neuf écoles, sélectionnées suivant la technique d'échantillonnage raisonné. Les critères ayant guidé ce choix ont été définis comme suit : la taille de la population autochtone dans les écoles, la disponibilité des listes des inscrits au titre des années scolaires 2017-2018, 20182019 et 2019-2020. Il s'agit des écolesMadzounou, Ingolo 2, Likouala, Sala Mbamba, Yomi, Tongo, Mbomo, Kengue et Bandize.Nous avons distingué quatre écoles majoritairement autochtones et cinq minoritairement autochtones. Le tableau 1 présente la répartition des élèves selon l'année scolaire et la classe pédagogique.

Tableau1. Données brutes des effectifs globaux des élèves par classe pédagogique et par année scolaire

\begin{tabular}{|c|c|c|c|c|c|c|c|}
\hline \multirow[b]{2}{*}{ Années } & \multicolumn{6}{|c|}{ Effectifs par classe pédagogique } & \multirow[b]{2}{*}{ Total } \\
\hline & CP1 & $\mathrm{CP} 2$ & CE1 & CE2 & CM1 & CM2 & \\
\hline $17-18$ & 199 & 111 & 85 & 50 & 25 & 12 & 482 \\
\hline $18-19$ & 291 & 142 & 67 & 37 & 23 & 13 & 573 \\
\hline $19-20$ & 304 & 157 & 54 & 41 & 18 & 8 & 582 \\
\hline Total & 794 & 410 & 206 & 128 & 66 & 33 & 1637 \\
\hline
\end{tabular}

Source: Inspection scolaire de Zanaga

Par ailleurs, en ce qui concerne les données qualitatives, un échantillon de convenance deonze (11) élèves décrocheurs autochtones a été constitué.

La collecte des données s'est déroulée en deux phases. La première a consisté à rassembler les rapports de rentrée dans les différentes écoles. Au cours de la deuxième phase les participants ont été entretenus individuellement à leur domicile. Il est utile de préciser que l'enquêteur commis à cette tâche a été formé préalablement à la conduite des entretiens. En effet, natif de Zanaga, il a réalisé ces entretiens en langue locale (Téké). Les entrevues ont été enregistrées à l'aide d'un dictaphone, puis transcrites et traduites, aux fins des différentes analyses.

Les analyses des données de l'étude documentaire ont porté sur la statistique descriptive et descriptive. A cet effet, nous avons utilisé le logiciel Mathlab. Celles issues des entrevues ont fait l'objet d'une analyse de contenu (Paillé et Mucchielli, 2010).

\section{RÉSUltats}

Nous présentons d'abord les résultats issus de l'étude documentaire, puis ceux des entrevues. 
Décrochage Et Vécu Scolaires Des Élèves Autochtones De La Circonscription Scolaire De Zanaga, République Du Congo

\subsection{Résultats De L’étude Documentaire}

Ces résultats concernent l'analyse globale du décrochage, la durée de vie scolaire et l'analyse comparée du décrochage dans les différents types d'écoles.

\subsubsection{Taux d'inscription des élèves autochtones au titre de trois années scolaires}

Les figures 1 et 2 suivantes représentent en pourcentage, par niveau d'étude et par cycle, les taux d'inscription des élèves autochtones, au cours des trois années scolaires retenues.

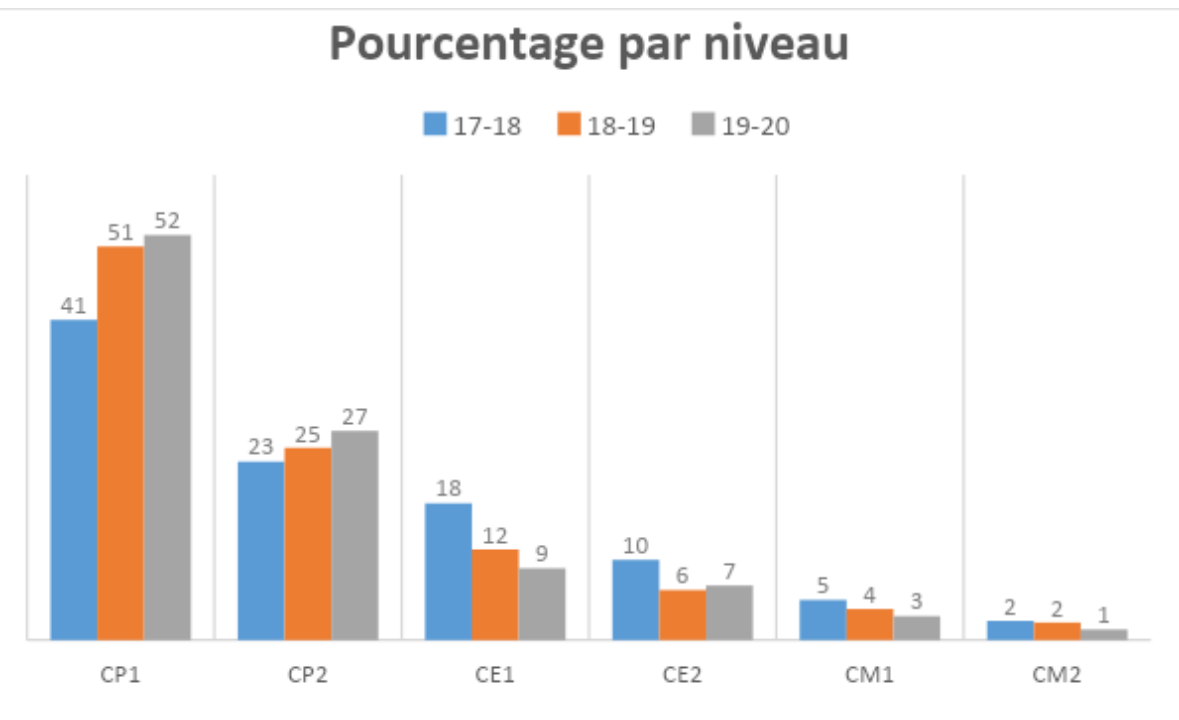

Figure1. Représentation des élèves autochtones en pourcentage niveau d'étude

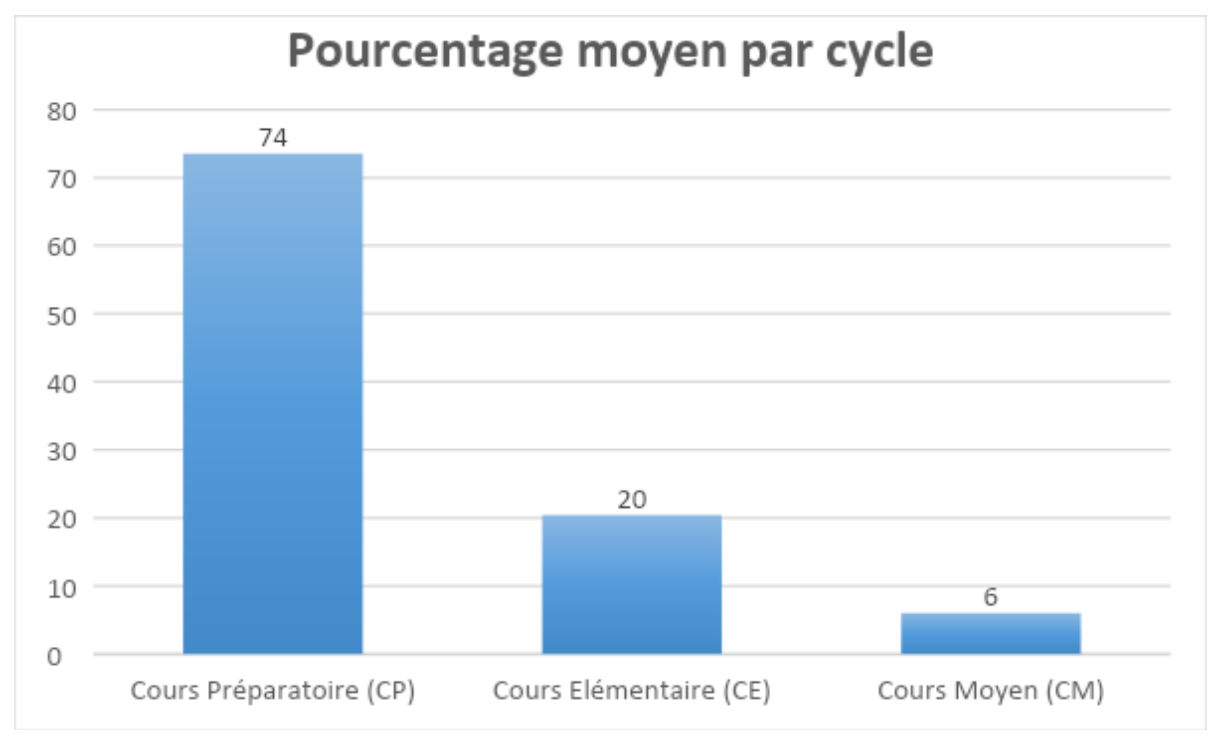

Figure2. Représentation des élèves autochtones en pourcentage par cycle étude

La lecture des données des figures 1 et 2 permet de constater quela tendance globale est à la croissance du nombre d'élèves scolarisés. Chaque année, les effectifs augmentent en CP1 et CP2, en moyenne $74 \%$ d'élèves sont au cours préparatoire. Cependant, ces effectifs décroissent dans les niveaux d'études supérieurs. (CE1, CE2, CM1 et CM2). Ce qui est une indication d'abandon significatif de la scolarité après le $\mathrm{CP} 2$;

\subsubsection{Durée de vie moyenne à l'école}

Nous avons déterminé la durée de vie moyenne à l'école primaire non pas en termes d'années de scolarisation, mais en termes de niveau de scolarisation. Pour ce faire, nous avons défini par :

- X : la variable aléatoire représentant la classe à laquelle on quitte le système scolaire. Elle peut prendre les valeurs $n=1,2,3,4,5.1$ représentant le $\mathrm{CP} 1$ et 5 le CM1. On considère que quitter le système scolaire au bout du CM2 est normal et donc sans objet dans le cadre de notre étude. 
Décrochage Et Vécu Scolaires Des Élèves Autochtones De La Circonscription Scolaire De Zanaga, République Du Congo

On s'est proposé de modéliser le processus étudié par une loi géométrique de paramètre p. Cette loi est appropriée pour étudier la durée de vie. La durée de vie moyenne est alors donnée par l'espérance de la loi qui est égale à $1 / \mathrm{p}$.

Plus précisément, on noté par :

- $\mathrm{p}$ : la probabilité d'abandonner.

- q : la probabilité de demeurer scolarisé. On peut noter que $\mathrm{q}=1$ - $\mathrm{p}$.

La probabilité de quitter le système scolaire au niveau $\mathbf{n}$ est donc $\mathrm{P}(\mathrm{X}=\mathrm{n})=\mathrm{pq}^{\mathrm{n}-1}$.

La probabilité de rester scolarisé après le niveau n est donc $\mathrm{P}(\mathrm{X}>\mathrm{n})=q^{n}=\frac{\sum_{l=n+1}^{6} E(i)}{\sum_{I=1}^{6} E(i)}$

Où E(i) est l'effectif du niveau i.

En recourant à une technique d'ajustement de courbes on peut donc identifier le paramètre q et en déduire $\mathbf{p}$.

On trouve $\mathrm{q}=0,48$ et donc $\mathrm{p}=0,52$.

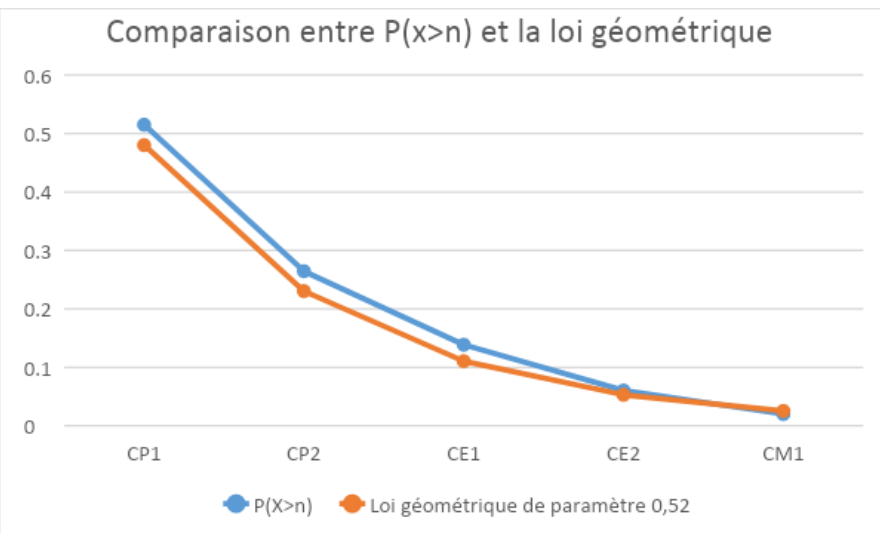

Figure3. Représentation des données expérimentales et la loi géométrique de paramètre 0, 52

$\mathrm{Au}$ regard de cette figure, il apparait que les données expérimentales et la loi géométrique sont quasiment identiques. Cette correspondance a été confirmée par le test statistique de KolmogorovSmirno, à une précision de $5 \%$. Les données expérimentales suivent donc une loi géométrique de paramètre 0,52 . De ce fait, on peut déduire que la durée de vie est égale à $n=1 / 0.52$, soit environ $n=2$, ce qui correspond à la classe de CP2.

\subsubsection{Comparaison des taux de décrochage entre les deux types d'écoles}

Nous avons d'abord analysé la baisse d'effectif d'une année à une autre en partant du CP1 au CP2 puis du CP2 au CE1. Les résultatssontreprésentés sur la figure 4 ci-dessous.

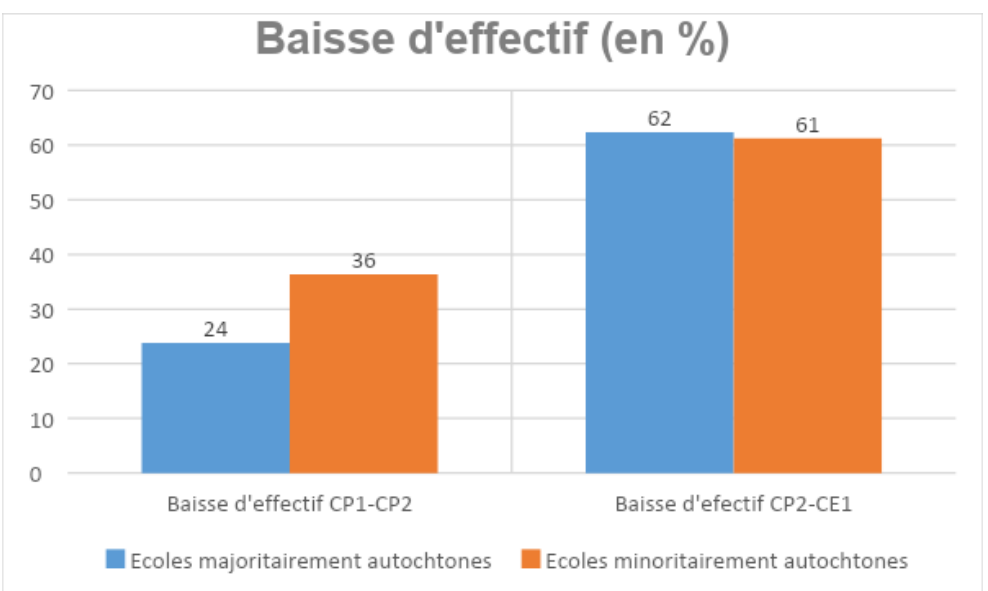

Figure4. Représentation de l'ampleur du décrochage selon le type d'écoles 
La lecture des données de cette figure montre que la différenciation entre type d'écoles semble se faire surtout dans la transition CP1-CP2. On peut aussi noter que d'après cette figure, le taux de décrochage en CP1 est d'au moins $24 \%$ dans les écoles majoritairement autochtones, tandis qu'il est d'au moins $36 \%$ dans les écoles minoritairement autochtones.

Ces résultats ont été complétés par des analyses du vécu scolaire.

\subsection{Résultats Des Entrevues}

L'analyse des récits du vécu scolaire des décrocheurs autochtones a permis de regrouper leurs propos autour de deux principaux thèmes: intégration scolaire et perception de l'école.

\section{$\checkmark$ Intégration scolaire}

La majorité des participants ont déclaré qu'ils ne se sentaient pas bien à l'école, ils n'avaient pas de bons rapports avec les Bantous. Un répondant s'est exprimé en ces termes: "Quand les frères n'étaient pas là, je ne pouvais pas parler avec les autres élèves Bantous ». Un autre a dit: "On ne s'occupait pas des 'toctocs" c'est comme si on n'existait pas...comme si on n'était pas aussi des personnes ».

Les participants ont aussi relevé l'atteinte de leur dignité et le manque de considération à leur égard. A ce sujet, un participant a déclaré ce qui suit: «Les Bantous nous insultaient, ils disaient qu'on était bête, j'avais honte et j'étais souvent triste... Pour ne pas avoir des problèmes avec eux, je restais seul quand mes frères n'étaient pas là... ».

Ce dénigrement a conduit certains participants à vouloir une école ayant davantage des élèves autochtones. Un participant s'est exprimé à propos : «Quand le maître nous mettait en groupes pour travailler, les élèves Bantous n'aimaient pas se mettre avec nous (...) Moi je voulais pour moi être avec les élèves 'toctocs'». Un autre a dit: « quand nous sommes entre nous, c'est vraiment bien... Pourquoi on n'aurait pas une école pour nous ?...».

L'intégration de ces élèves dans le milieu scolaire a été également analysée au travers de leurs rapports avec les enseignants.

En effet, il semble que cette relation se résume en termes de maître-élève, c'est-à-dire confinés dans la sphère didactique (en classe), car les participants ont mentionné que les échanges entre élèves autochtones et l'enseignant étaient rares. Deux participants l'ont exprimé en ces termes: "Les enseignants n'étaient pas souvent à côté de moi ». Un autre répondant a déclaré que: «Le maître aime seulement les élèves Bantous, pas nous ». " Le maître n'écoute que les élèves intelligents, il ne passe pas du temps avec nous quand on ne comprend pas à faire quelque chose (....) ils ne sont pas proche de nous quand ils nous apprennent des choses de l'école ... Quand un 'toctoc' faisaient une faute en classe le maître le punissait ».

\section{$\checkmark \quad$ L'école Vue Par Les Élèves Autochtones}

A la question de savoir comment l'école était-elle perçue par les décrocheurs autochtones, il ressort des analyses des verbatim qu'ils ne voyaient pas l'obligation d'aller à l'école. Parce que le fait d'être scolarisé ne changeait pas leurs conditions sociales et économiques. En plus, l'école n'est pas un milieu favorable pour leur épanouissement. Quatre participants l'onexprimé comme suit:

- «Pourquoi nous devons aller à l'école, nous les 'toctocs' nous n'allons jamais travailler dans les bureaux comme les bantous (D1).

- «L'école n'est pas pour nous...mon père me disait souvent que partais pour passer le temps alors que je devrais aller avec lui pour apprendre comment trouver à manger...»(D2).

- «Mon frère était aussi à l'école, mais il fait quoi avec ce qu'il a appris ça ne sert à rien (...) pourquoi alors souffrir là où on ne vous aime pas, où on rit de nous... »(D3).

- «Moi je ne comprenais rien de tout ce qui se passait en classe, je suis allée pendant une année et puis j'ai quitté (...) Ce que j'ai trouvé difficile, c'est parler leur langue, on parle des choses difficiles et j'ai décidé de rester à la maison » (D4).

\section{DISCUSSION}

Ce cadre de référence visait deux objectifs, à savoir, faire un état des lieux du décrochage scolaire chez les enfants autochtones et analyser leur vécu scolaire en vue de suggérer des pistes d'intervention 
Décrochage Et Vécu Scolaires Des Élèves Autochtones De La Circonscription Scolaire De Zanaga, République Du Congo

susceptibles de favoriser leur maintien à l'école. Pour ce faire, nous avons réalisé une enquête de terrain au moyen de l'étude documentaire et des entrevues. Dans cette partie de notre élaboration, nous synthétisons et discutons les résultats obtenus à la lumière des travaux de recherche antérieurs.

Il ressort des analyses documentaires que l'ampleur du décrochage est toujours significatif (figures 1 et 2), malgré les stratégies d'incitation et de maintien à l'école mise en place. Aussi, le phénomène est plus accentué dans les écoles où les autochtones sont minoritaires (figure 4). Ces résultats s'apparentent à ceux de Bazylak (2002) qui a affirmé, dans une étude de cas sur la persévérance scolaire aux études secondaires, que lorsque les étudiants autochtones sont dans des milieux où ils sont plus nombreux, ils unissent leur voix, se soutiennent et se renforcent les uns aux autres.

Ces résultats suggèrent que la coloration de l'environnement scolaire a un impact prégnant sur le maintien ou non des apprenants à l'école. Cette hypothèse nous paraît plausible, au regard des résultats réalisés dans les écoles ORA (Observer, Réfléchir, Agir), implantées dans le département de la Sangha et de la Likouala. Ce projet qui s'inscrit dans un programme national de scolarisation des autochtones, est une initiative des prêtres spiritains de l'église catholique. L'objectif est d'accompagner les enfants pygmées pour intégrer le système éducatif formel. Ceux-ci sont formés pendant deux ans, à l'issus desquels ils sont censés rejoindre la classe de CE1. Il sied de signaler que les instructeurs de ces écoles sont issus de la communauté autochtone. Ils utilisent la langue maternelle dans leur pédagogie adaptée. Fort de cette expérience, $80 \%$ d'enfants des écoles ORA ont été scolarisés à l'école formelle et ont été maintenus jusqu'à l'achèvement du cycle primaire.

Dans le même sens, plusieurs études ont montré que les étudiants des Premières Nations qui fréquentent des établissements scolaires de culture occidentale réussissent davantage si ces milieux scolaires intègrent des éléments de leur culture première (Assemblée des Premières Nations, 2010a; Canada MillenniumScholarshipFoundation, 2005; Conseil canadien sur l'apprentissage, 2009).

Aussi, Malatest et al., (2002) rapportent-ils sur l'éducation postsecondaire des étudiants autochtones qu'un des éléments favorisant leur décrochage est lorsqu'ils perçoivent leur milieu universitaire comme un milieu impersonnel et intimidant, dans lequel leurs cultures, traditions et valeurs ne sont pas reconnues. Dans la même optique, Timmons (2009) dans ses travaux de recherche portant sur la rétention des étudiants autochtones aux études postsecondaires a relevé que les centres de ressources destinés spécifiquement aux étudiants autochtones étaient considérés comme des facilitateurs importants pour leur réussite scolaire et leur adaptation sociale.

Dès lors, on peut s'interroger sur la possibilité de réalisation d'un cycle primaire complet par ces élèves. Notre réponse est affirmative, à condition d'incorporer dans les programme de formation les éléments de leur culture. Effectivement selon la théorie historico- culturelle de Vygotsky (1985), il y a apprentissage lorsque l'apprenant peut incorporer ce qui vient de l'autre personne en y donnant un sens dans son histoire. Autrement dit, il y a apprentissage si ce qui est proposé n'est pas trop éloigné de ce que l'apprenant connait déjà. Cette façon de concevoir l'apprentissage demande que l'élève soit placé dans des situations concrètes et qu'il ait l'opportunité de revenir sur son expérience avec ses pairs ou son enseignant. C'est par cette rétroaction qu'il pourra structurer ses nouvelles connaissances.

On peut alors s'interroger sur l'importance de la culture d'origine dans la scolarisation et le maintien de ces élèves à l'école.

En effet, au cours de nos entretiens, les participants ont mentionné le mal être ressenti dans cette nouvelle structure.La langue d'enseignement leur était nouvelle, celle-ci ne facilitait pas les apprentissages. Ils se sentaient étranger dans ce milieu, faute d'éléments de leur communauté d'appartenance. Nos résultats corroborent ceux de Rodon (2008) qui a constaté dans ses travaux de recherche que les étudiants autochtones admis dans les universités générales, ne se sentent pas à l'aise d'utiliser les ressources existantes à l'université. Ces étudiants, faute de leurs éléments culturels, ressentaient un manque de reconnaissance.

De ce qui précède, nous présumons que les éléments culturels sont un gage d'adaptation et de réussite scolaire pour les enfants autochtones.

En dehors des questions relatives à l'inadaptation scolaire, nous avons relevé la perception négative que les élèves décrocheurs ont de l'école. Pour expliquer cette attitude, plusieurs raisons peuvent être évoquées. Il peut s'agir d'un manque d'espoir quant à l'utilité de la formation, à la nature des interactions entre l'apprenant et l'institution... 
A propos de la première raison, si nous admettons que l'école est la clé du développement socioéconomique, cette hypothèse est loin d'être validée pour les peuples autochtones. En effet, de leur point de vue, rien ne garantit un avenir professionnel pour eux, dans le contexte social congolais. Par conséquent, l'école ne peut être perçue par eux comme une nécessité.

Cette perception restrictive de l'école est à notre avis, un facteur potentiel d'amotivation. Les enfants autochtone se retirent de l'activité qu'ils pratiquent, parce qu'ils se sentent désabusés, se demandant quelque fois à quelle fin ils vontà l'école (Vallérand et al.,2001). En plus, au cours des entretiens, nous avons relevés une deuxième constante, notamment la perception que les autochtones ont d'euxmêmes. En effet, les répondants, en se comparant aux élèves Bantous, ont affiché une image négative d'eux. Ils déclarent ne pas être à leur place parmi leurs pairs Bantous. En d'autres termes, ils éprouvent un sentiment de marginalisation, une dévalorisation de soi. Ce qui les amène indubitablement à montrer peu d'enthousiasme et de motivation à fréquenter l'école.

Or, selon les théories de l'autodétermination de Bandura (2003, p.12) pour réaliser et réussir une activité, l'individu doit croire en ses capacités. Pour l'auteur, ce sont ces croyances d'efficacité personnelle qui régulent les aspirations, les choix de comportement, la mobilisation, la poursuite de l'effort, ainsi que les réactions émotionnelles. A la lumière de cette théorie, nous présumons que les enfants autochtones ont un faible sentiment d'efficacité personnelle. Ce qui justifie le peu de raison d'agir ou de persévérer face aux difficultés scolaires rencontrées.

Enfin, la troisième constante à laquelle nous attribuons le refus de l'école par les enfants autochtones est la qualité des rapports interpersonnels. Il est connu que les croyances, les attitudes et les comportements des enseignants sont des variables qui produisent des effets substantiels sur la relation enseignant-élèves et sur la motivation des élèves dans leurs apprentissages (Davis, 2003). Dans le cadre de cette étude, nous avons relevé que ces relations, telles que décrites par les participants sont froides.

En effet, de leur avis, l'école est tissée de préjugés, un lieu d'humiliation, de discrimination...Les participants ont déploré l'usage par les enseignants des mots dégradants pour les désigner, des injures lorsqu'ils se trouvent dans l'incapacité de répondre aux exigences scolaires....Nous présumons que les gestes ou les paroles de dépréciation de la part des enseignants ont eu un impact important sur l'engagement de ces élèves et sur leurs attitudes à l'endroit de l'école.

Cette étude qui n'a pas la prétention d'avoir circonscrit toutes les questions relatives à cette problématique, présente des limites. La première se rapporte aux caractéristiques particulières de l'échantillon utilisé. En effet, la taille de l'échantillon empêche la généralisation des résultats à tous les décrocheurs autochtones. Il serait par conséquent intéressant d'effectuer des recherches sur un échantillon plus large.

\section{CONCLuSion}

Ce présent travail de recherche, a tenté d'apporter un éclairage sur la question du décrochage scolaire des enfants autochtones. Les objectifs poursuivis à cet effet ont été de deux ordres, précisément faire un état des lieux du décrochage et requérir les opinions des décrocheurs autochtones concernant leur expérience scolaire. Pour répondre à ces questions, nous avons réalisé une étude documentaire et des entrevues semi-dirigées. L'analyse descriptive et inférentielledes effectifs des élèves scolarisés au cours de trois années scolaires a permis de constater que, malgré les stratégies incitatives et de maintien à l'école mise en œuvre par les pouvoirs publics, avec l'aide des partenaires sociaux à l'éducation les taux de décrochage demeurent élevés. Le phénomène est plus accentué dans les écoles où les autochtones sont minoritaires. La durée de vie scolaire quant à elle est très éphémère, autrement dit, la probabilité qu'un élève reste à l'école est de deux années. Aussi, l'analyse de contenu des expériences scolaires vécues par ces élèves a révélé des perceptions négatives de l'école et des mauvais rapports interpersonnels.

De ce qui précède, dans la perspective d'une inclusion scolaire effective des enfants autochtones, il est impératif que les recherches soient orientées en direction des stratégies efficientes à mettre en place, en vue de rendre l'espace d'apprentissage scolaire plus attrayant. Il s'agit entre autre de repenser les programmes de formation, de sensibiliser davantage les populations autochtones sur l'impact de l'éducation, de promouvoir le vivre-ensemble entre les peuples autochtones et Bantous et de vulgariser l'expérience des écoles ORA. 
Décrochage Et Vécu Scolaires Des Élèves Autochtones De La Circonscription Scolaire De Zanaga, République Du Congo

\section{RÉFÉRENCES BIBLIOGRAPHIQUES}

[1] Ballion, R., (2000). Les conduites déviantes de lycéens. Paris, Hachette.

[2] Bandura, A., (2003). Autoefficacité : le sentiment d'efficacité personnelle. Bruxelles: De Boeck.

[3] Bazylak, D., (2002). Journeys to Success Perceptions of Five Female Aboriginal High School Graduates.Canadian Journal of Native Education, 26 (2), 134-151.

[4] Blaya, C., (2010a). Décrochages scolaires : L'école en difficulté. Bruxelles : De Boeck

[5] Blaya, C., (2010b). «Décrochage scolaire: Parents coupables, parents décrocheurs ? . Informations sociales, $\mathrm{n}^{\circ} 161, \mathrm{p} .46-54$.

[6] Blaya, C., et Hayden, C. (2003). Constructions sociales des absentéismes et décrochages scolaires en France et en Angleterre, LARSEF/Observatoire européen de la violence scolaire.

[7] Bronfebrenner, U., (1993). The ecology of cognitive development: research models and fugitive findings. In R. H. Wozniaket K. W. Fischer (Eds.). Development in context: acting and thinking in specific environnemts (p-p 3-44). Hillsdale : Lawrence Erlbaum Associates Inc.

[8] Canada Millennium Scholarship Foundation, (2005).

[9] Conseil canadien sur l'apprentissage, (2009).

[10] Conseil canadien sur l'apprentissage. (2007). Redéfinir le mode d'évaluation de la réussite de l'apprentissage chez les Autochtones. Page consultée le 19 mai 2015 de http://www.cclcca.ca/CCL/ Reports/RedefiningSuccessInAboriginalLearning/RedefiningSuccessModels-2.html.

[11] Davis, H.A., (2003). Conceptualizing the role and influence of student-teacher relationships one children's social and cognitive development. Educational psychologist. 38(4), pp 207-234.

[12] Déclaration universelle des droits de l'homme en 1948.

[13] Douat, E.,(2011). L'école buissonnière. Paris : La Dispute.

[14] Favresse, D. et Piette, D., (2004). Les jeunes en marge du système scolaire: inscription dans une socialisation de l'exclusion. L'observatoire, 43, 87-91.

[15] Fortin, L. Royer, E., Marcotte, D., Potvin, P.etYergeau, E., (2004). La prédiction du risque de décrochage scolaire au secondaire: facteurs personnels, familiaux et scolaires. Revue Canadienne des Sciences du Comportement, 36(3), 219-231.

[16] Gauthier, R., (2005). Le rapport à l'institution scolaire chez de jeunes amérindiens en fin de formation secondaire : contribution à la compréhension du cheminement scolaire chez les Autochtones. Thèse de doctorat inédite, Université du Québec à Chicoutimi.

[17] Glasman, D., (2011). Le décrochage scolaire. Paris, Presses universitaires de France.

[18] Glasman, D.,Oeuvrard. F., (2004).La déscolarisation, Paris, La dispute.

[19] Itoua-Yoyo, A., (2008). Stratégie nationale d'éducation des populations autochtones du Congo (SNEPAC).

[20] Janosz, M., Le Blanc, M., Boulerice, B. et Tremblay, R. E., (2000). Predicting different type of school dropouts : A typological approach with two longitudinal samples. Journal of Educational Psychology, 92(1), 171-190.

[21] Kama-Niamayoua, R., (2008). La scolarisation des enfants issus des familles des peuples autochtones. Communication à la $48^{\text {ème }}$ session de la Conférence internationale de l'éducation, www.ibe.unesco.org/ fileadmin/user_upload/Policy_Dialogue/

[22] Kitsimbou, X., (2020). Evaluation de la situation des populations autochtones au Congo Brazzaville HAL Id: hal-02878981 https://hal.archives-ouvertes.fr/hal-02878981.

[23] Kono, M. et Tangu, S., (2013). L'éducation chez les peuples autochtones : Baka, Bagyeli,Bakola, Bedzang. (2013). www.ced.cam.org.

[24] Lee, V.-E., Burkam, D.-T., (2003). Dropping out of high school: The role of school organization and structure. AméricanEducationalResearch Journal, $\mathrm{n}^{\circ} 40$ (2) 353-393.

[25] Loi $\mathrm{n}^{\circ}$ 5-2011 du 25 février, portant promotion et protection des droits des populations autochtones.

[26] Mafoukila, C. M., (2006). La Scolarisation des enfants pygmées au Congo. Presses Universitairesd'Afrique.

[27] Malatest, R. A. Associates Ltd et The Council of Ministers of Education Canada (CMEC). (2002). Best Pratices in Increasing Aboriginal Post-secondary Enrolments Rates.

[28] ONU, (1959).Déclaration des droits de l'enfant.

[29] Paillé, P., et Mucchielli, A., (2012). L'analyse qualitative en sciences humaines et sociales.(3e éd.). Paris, France : Éditions Armand Colin.

[30] Centre National de la Statistique et Etude Economique (CNSEE) : Populations autochtones du congo www.cnsee.org/index.php?option=com_content \&view=article 
Décrochage Et Vécu Scolaires Des Élèves Autochtones De La Circonscription Scolaire De Zanaga, République Du Congo

[31] Presseau, A., Martineau, S., Bergevin, C., et Dragon, J.- F., (2006). Contribution à la compréhension du cheminement et de l'expérience scolaires de jeunes autochtones à risque ou en difficulté en vue de soutenir leur réussite et leur persévérance scolaires. FQRSC.

[32] Rapport des Nations Unies (2014), Situation de la population mondiale en 2014.

[33] Rodon, T., (2008). Les étudiants autochtones à l'Université Laval : enquêté sur les besoins et les problématiques. Les cahiers du CIERA, Université Laval, Quebec, QC.

[34] Timmons, V., (2009). Retention of Aboriginal Students in Post-Secondary Institutions in Atlantic Canada: An Analysis of the Supports Available to Aboriginal Students. Montréal,

[35] Canada : Conseil canadien sur l'apprentissage.

[36] UNESCO, 1994;Déclaration de Salamanque et cadre d'action pour l'éducation et les besoins spéciaux.

[37] Vallerand, R. J., Pelletier, L., Fortier. M. et Briere, N.M., (2001). Association between perceived autonomy support, forms of self- regulation and persistence study. Montréal. Motivation and Emotion

[38] Vygotsky, L.S., (1985). Pensée et langage (trad. F. Sève). Paris : Editions Sociales.

\section{AUTHOR's BIOGRAPHY}

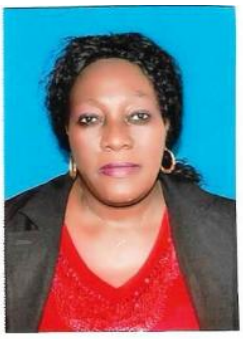

Yvette BakinguBakibangou, Docteur en Sciences de 1'Education, Enseignante chercheure à l'Institut Supérieur d'Education Physique et Sportive (Université Marien Ngouabi)

Citation: Yvette BakinguBakibangou, Samuel Mawete. "Décrochage Et Vécu Scolaires Des Élèves Autochtones De La Circonscription Scolaire De Zanaga, République Du Congo" International Journal of Humanities Social Sciences and Education (IJHSSE), vol 8, no. 2, 2021, pp. 48-58. doi: https://doi.org/10.20431/2349-0381.0802006.

Copyright: (c) 2021 Authors. This is an open-access article distributed under the terms of the Creative Commons Attribution License, which permits unrestricted use, distribution, and reproduction in any medium, provided the original author and source are credited. 